\section{Antrostomía ósea lateral simplificada. Reporte de caso.}

Lateral bone simplified antrostomy. A case report.

\section{Resumen}

Este reporte clínico presenta un procedimiento quirúrgico simplificado para el acceso al antro del seno maxilar a través del abordaje lateral, lo que reduce el potencial de perforación de la membrana del seno y las complicaciones posteriores cuando los materiales del injerto y los implantes dentales se colocan en el seno. Debido a las limitaciones visuales, las perforaciones y las complicaciones asociadas pueden poner en peligro la tasa de éxito del injerto y los implantes. Si bien existe una falta de datos clínicos, las observaciones clínicas sugieren que el procedimiento, descrito por los autores como antrostomía del hueso por cepillado lateral, puede reducir la posibilidad de perforación de la membrana del seno maxilar durante los abordajes laterales para el injerto del piso del seno maxilar. La técnica consiste en la utilización de instrumentos especialmente diseńados rotatorios que desgastan el hueso en capas más delgadas, con menos posibilidades de eliminar el exceso de hueso y la perforación de la membrana. El objetivo de la publicación es mostrar la secuencia clínica del procedimiento quirúrgico. Se concluye que la antrostomía ósea lateral simplificada es sencilla de realizar y disminuye la aparición de complicaciones. Palabras Clave: Implantes dentales, Seno Maxilar, cirugía bucal.

\section{Abstract}

This clinical report presents a simplified surgical procedure for accessing the maxillary sinus antrum via lateral approach, which reduces the potential for sinus membrane perforation and subsequent complications when graft materials and dental implants are placed into the sinus. Due to visual limitations, perforations and associated complications can jeopardize the success rate of the graft and the implants. While there is a lack of clinical data, clinical observations suggest that the procedure, described by the authors as lateral bone planing antrostomy, can reduce the possibility of perforation of the maxillary sinus membrane during the lateral approach to the grafting of the maxillary sinus floor. The technique involves the use of specially designed rotary instruments that plane away the bone in thinner layers, with less chance of excess bone removal and membrane perforation. The aim of the publication is to show the clinical sequence of the surgical procedure. We conclude that the simplified lateral bone antrostomy is simple to perform and reduces the appearance of complications.

Key Words: Dental Implants, Maxillary Sinus, oral surgery
Casos Clínicos

\section{Henry Vilcapoma Guerra ${ }^{1}$, Elmer Salinas Prieto ${ }^{2}$}

\footnotetext{
${ }^{1}$ Cirujano Dentista, egresado de la Universidad Nacional Mayor de San Marcos

${ }^{2}$ Jefe del Servicio de Periodoncia del Departamento de Salud Oral de la Fuerza Aérea del Perú
}

Correspondencia:

CD Henry Vilcapoma Guerra

Av. José Santos Chocano 338 Urb. Villa Los Angeles. Los Olivos

Teléfono:(511)521-4979

E-mail: henry_vilcapoma@hotmail.com

Fecha de recepción: 17-10-11

Fecha de aceptación: 26-11-11

\section{Introducción}

El tratamiento con implantes en la zona posterior del maxilar superior plantea diversos problemas. Frecuentemente hay disponibilidad ósea vertical insuficiente para la colocación de implantes debido a la reabsorción de la cresta alveolar que a veces se une a un aumento de la neumatización del seno maxilar tras la extracción de las dientes dentarias de ese sector. Además, es la zona con mayor prevalencia de hueso tipo IV (hueso con escaso componente cortical y abundante componente esponjoso), según la clasificación de Lekholm y Zarb. ${ }^{1-15}$

Los métodos quirúrgicos más importantes incluyen los injertos sinusales para incrementar la altura ósea disponible, los injertos onlay para aumentar la anchura ósea y abordajes quirúrgicos modificados para insertar los implantes en zonas con menor densidad ósea. El injerto en el seno maxilar para resolver el problema de una menor altura ósea vertical se ha convertido en un procedimiento popular y predecible en las últimas décadas. 1,2, 5, 8, 9, 10, 16, 17

Las técnicas quirúrgicas básicas para el injerto en el seno maxilar son: un abordaje transalveolar o técnica cerrada (originalmente descrito por Summers) o un abordaje lateral o técnica abierta (similar al adoptado para la radicalización del seno maxilar según Caldwell - Luc, descrito por Tatum). 1,5,7,8

La anatomía del seno es variada, conformada generalmente por una cavidad única, pero puede estar tabicado y aumentar las dificultades en caso de necesitar hacer un relleno. Su interior está tapizado por un epitelio ciliado con células que producen una secreción serosa y mucosa, cuya función es limpiar atrapando gérmenes y polvo ambiental para eliminarlos hacia la nariz a través de un agujero llamado ostio, que está en el meato medio, por lo tanto, el drenaje no puede hacerlo por gravedad debido a que está más alto que la base y necesita de las ondas que provocan las cilias. $1,2,4,5$

Dentro de los estudios radiográficos, es posible utilizar: radiografías intraorales, radiografía panorámica (ortopantomografía), telerradiografía de cráneo en proyección lateral y tomografía computarizada. ${ }^{11}$

La tomografía es el único estudio que permite conocer tridimensionalmente las morfología del seno, además de permitir evaluar las medidas exactas de los remanentes óseos y los tabiques, que es una de las mayores dificultades que se presentan, como así también el estado de salud del seno, porque es común encontrar hipertrofias inflamatorias, agudas o crónicas generalmente rela- 
cionadas con filtraciones bacterianas de dientes dentarias adyacentes o bien de sinusitis alérgicas crónicas. ${ }^{1,2,7,11,12}$

La clasificación de las diferentes técnicas de elevación de piso de seno maxilar depende de la altura de hueso residual entre la cresta alveolar y el piso de seno, descrito por Misch. 1, 2, 7, 8, 9, 15, 18, 19

En la técnica abierta o abordaje lateral para la elevación del piso de seno maxilar el abordaje se practica a través de una ventana lateral (acceso tipo Caldwell-Luc). Se realiza un colgajo a espesor total mediante una incisión supracrestal que permita delimitar la pared lateral del seno maxilar. Una vez delimitada esta estructura se realiza una "ventana quirúrgica" que se perfila a través de una osteotomía con fresa redonda de diamante. Una vez que se insinúa la membrana sinusal a través de la osteotomía, se procede a fracturar hacia adentro la "ventana quirúrgica" de forma que su arista superior actúe como una bisagra, trasladando hacia apical el nuevo suelo del seno maxilar. Durante este delicado procedimiento hay que procurar no perforar la membrana de Schneider, que se debe ir despegando lentamente mediante instrumentos especialmente diseñados para este propósito, para que así acompańe a la ventana ósea y quede incluida en el espacio de la nueva configuración del seno maxilar.

Una vez desplazada la membrana ósea con la membrana de Schneider se procede a colocar el material de relleno dentro del seno maxilar, simultaneando o no, según la situación clínica, la colocación de los implantes. Finalmente, y antes de proceder a la sutura del colgajo, se aislará la cavidad quirúrgica por medio de una membrana barrera, que excluya las células del compartimento conectivo procedente del colgajo.

La técnica traumática se divide, según se simultanee o no a la colocación del implante, en:

* Inmediata: colocación del implante al mismo tiempo que se realiza la elevación del seno.

* Diferida: colocación del implante un mínimo de 6 meses después de realizada la elevación del seno.La elección de una u otra técnica debe basarse en la altura ósea preoperatoria disponible y en la posibilidad de colocar los implantes con estabilidad primaria. ${ }^{18,19}$

El objetivo de la publicación es mostrar la secuencia clínica de un procedimiento de antrostomía ósea lateral simplificada en un paciente.

\section{Reporte del caso}

Paciente masculino de 43 años de edad, sin enfermedad sistémica, ni antecedentes patológicos personales. Acude a la consulta para una rehabilitación oral integral. En el examen clínico se observó un reborde edéntulo parcial superior unilateral (lado derecho) a nivel del diente 16. Fue solicitada una radiografía panorámica (Fig. 1) y una Tomografía Axial Computarizada (TAC) (Fig. 2) en la cual fue observada dimensiones óseas desfavorables para el diente 16 (altura de $6.9 \mathrm{~mm}$ y ancho promedio de $6.6 \mathrm{~mm}$ ).
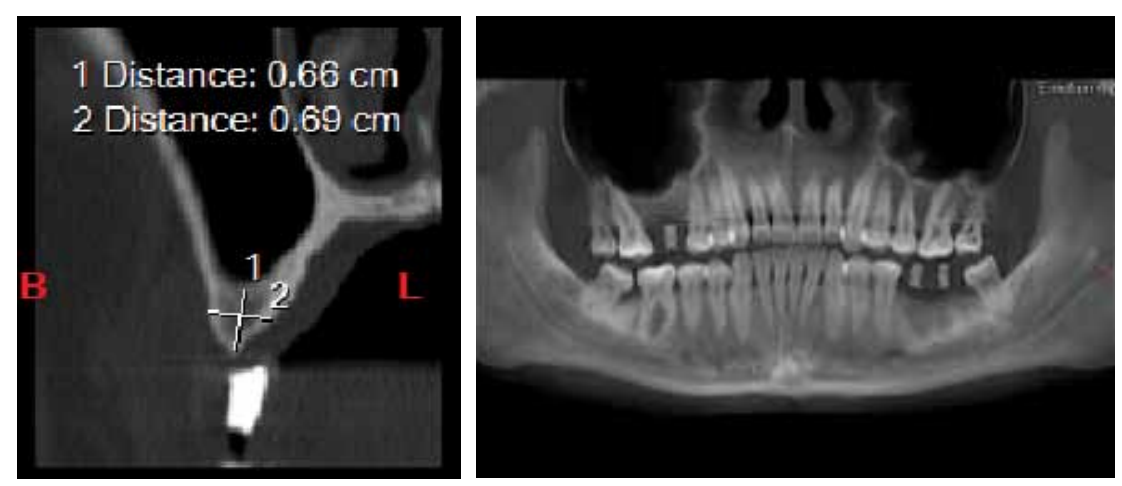

Fig 1. Radiografía Panorámica

Fig 2. Corte seccional a nivel del diente 16

El plan de tratamiento propuesto fue la colocación de un injerto sinusal con abordaje en la pared lateral y colocación simultánea de un implante dental.

La intervención quirúrgica se realizó con anestesia local (articaína al 4\% con epinefrina 1:100 000) se hizo una incisión de espesor total de forma trapezoidal, decolado de periostio y tejido conjuntivo. (Fig. 3). Se empezó a desgastar la pared exterior del seno maxilar hasta observar el límite de la membrana sinusal con una fresa DASK No. 5 montado sobre una pieza de mano de baja velocidad, bajo irrigación constante con solución fisiológica estéril (Fig. 4). Luego, se procedió con separadores curvos especiales al clivaje de la mucosa sinusal del piso y de las paredes, mesial y distalmente al acceso. (Fig. 5). Se preparó la zona implantar para el implante dental a nivel del diente 1.6 (Fig. 6), se colocó el material de relleno en la cavidad antral (matriz ósea desmineralizada con hueso esponjoso) (Fig. 7). Se colocó el implante dental en $1.6(4.5 \mathrm{X} 13 \mathrm{~mm})$ (Fig. 8). Se reposicionó el colgajo, finalmente se suturó por planos y con vycril (3.0) (Fig. 9).

Se indicó analgésicos: Dexametazona $4 \mathrm{mg}$ (amp), Ketorolaco 60mg (amp),

Naproxeno 550mg (comp).

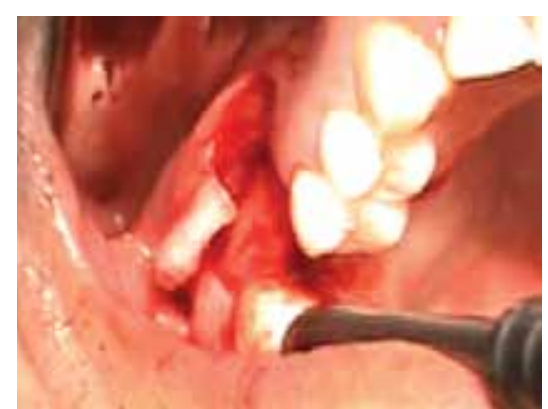

Fig 3. Colgajo de espesor total

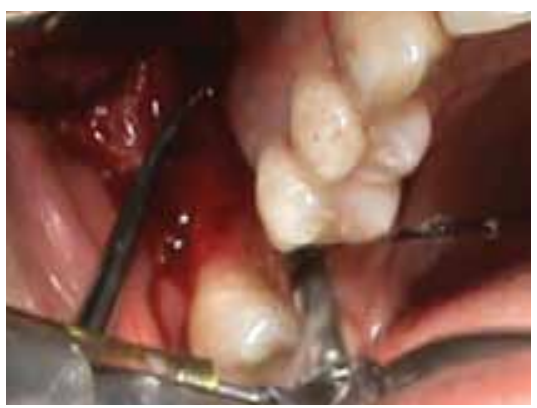

Fig 5. Separación de la membrana sinusal con cureta

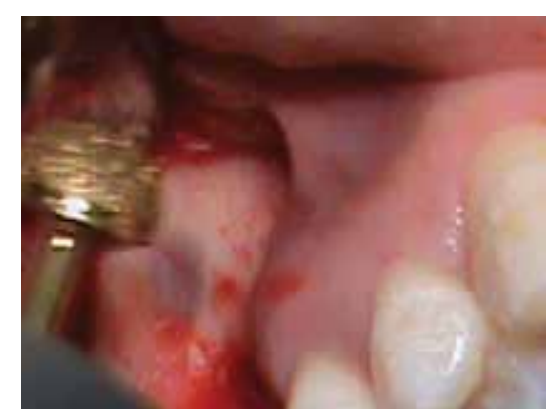

Fig 4. Fresado externo con DENTIUM DASK No. 5

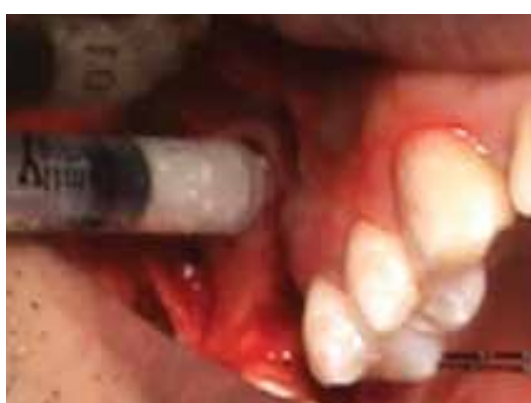

Fig 6. Osteotomía para colocación del implante 


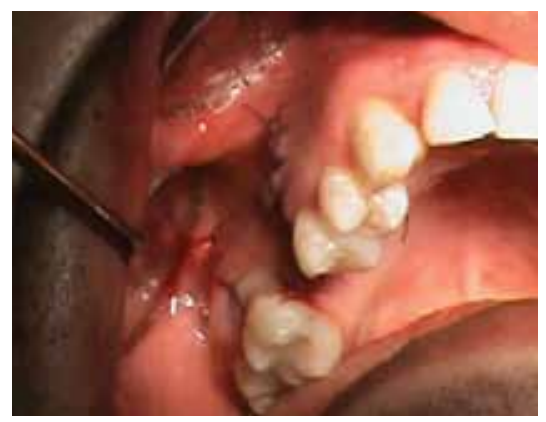

Fig 7. Inserción del relleno óseo

\section{Procedimiento clínico}

Se decidió aplicar incrementos de resina compuesta sobre las restauraciones anteriores sin la colocación de ácido y de adhesivo (técnica de Mock Up) con el fin de mejorar los anchos de las piezas comprometidas así como los ángulos de contacto interdental. Una vez obtenidas las características deseadas, se confeccionó una guía de silicona con masa pesada de las superficies palatinas directamente en boca (Fig. 3). Con aislamiento absoluto del sector anterosuperior; se realizó la remoción de las restauraciones antiguas y la eliminación del tejido cariado; se pulieron las superficies comprometidas y se biseló la preparación a nivel vestibular. Se aplicó ácido ortofosfórico al $37 \%$ sobre las superficies preparadas, 15 segundos en dentina y 30 segundos sobre esmalte; se lavó con abundante agua y se secaron las superficies con papel absorbente sin resecar las superficies. Se aplicó dos capas de un adhesivo de quinta generación (Fig. 4) y se frotó hasta evaporar el solvente del adhesivo, posteriormente se fotocuró por 20 segundos. Se colocó la guía de silicona confeccionada en boca y se procedió a la estratificación por capas anatómicas aplicando una capa de resina translucida que nos servirá como esmalte palatino (Fig. 5), se fotocuró el incremento de resina compuesta por 20 segundos. Posteriormente se aplicó una resina opaca de color A2 para reemplazar a la dentina y en el borde incisal colocamos una resina de cuerpo de color A1 para dar el efecto de línea alba del borde incisal (Fig.6) y se fotocuró los incrementos de resina compuesta por 20 segundos. Se aplicó un incremento de resina translucida en los espacios dejados al colocar la resina de dentina para dar un mayor efecto de translucidez. Se colocó una resina compuesta para esmalte de color A1 que cubra toda la cara vestibular que nos va a simular el esmalte vestibular y se fotocuró por 20 segundos. (Fig. 7). Posteriormente se retiró el aislamiento absoluto y con una fresa multilaminada hacemos los retoques de las restauracio-

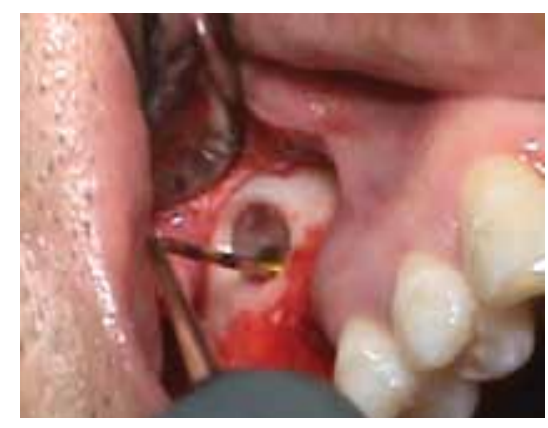

Fig 8. Colocación del implante dental

nes, controlamos la oclusión y posteriormente se dio el acabado y pulido a las restauraciones (Fig. 8).

\section{Discusión}

Dentro del diagnóstico se corrobora lo señalado por la mayoría de autores que confirman la pérdida de altura del reborde alveolar de la región postero superior, como la característica más resaltante, tras la pérdida de dientes dentarias en ese sector. ${ }^{1-15}$

Respecto a los exámenes complementarios, se confirma la gran importancia de la Tomografía Axial Computarizada (TAC) para el estudio de las dimensiones óseas (altura y grosor de hueso), calidad ósea y la evaluación de la anatomía de los senos maxilares. $1,2,7,11,12$

Siguiendo los criterios de la mayoría de autores, al tener una altura de hueso residual entre 4 a $6 \mathrm{~mm}$ a nivel de la diente 2.4 se procedió a realizar el levantamiento del piso del seno maxilar y colocación inmediata de los implantes, ya que con esas dimensiones se puede conseguir la estabilidad primaria. ${ }^{1,2,5,7,9,11,12,13,14,15,16,18,19,20}$

En la actualidad existen técnicas quirúrgicas de elevación del piso del seno maxilar que tienen como base las técnicas originales de Summers y Tatum, pero que se valen de ciertos instrumentos especiales para lograr separar cuidadosamente la membrana de Schneider como: balones de aire, hisopo de algodón con lidocaína, endoscopio, aparato de ultrasonido, etc. ${ }^{20-27}$

Por otro lado, algunos autores refieren que los resultados de los implantes dentales colocados con aumento de piso de seno maxilar fueron similares que aquellos sin elevación del piso de seno maxilar. ${ }^{23-25}$

Una de las grandes variantes respecto a los injertos sinusales es el tipo de injerto óseo a colocar en la cavidad subantral confeccionada. Diversos autores han realizado estudios respecto a este tema, la mayoría concluye que el mejor material es el hueso autólogo o autoinjerto

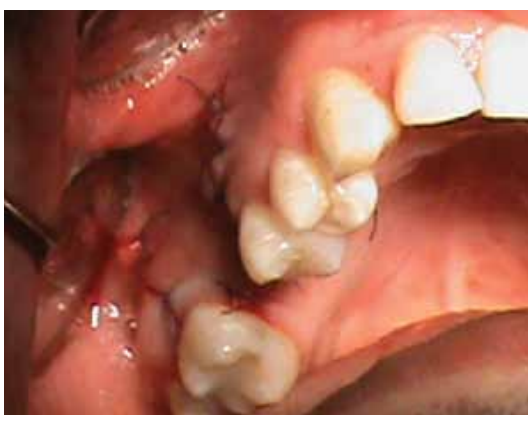

Fig 9. Sutura

(derivado del propio paciente). Sin embargo, los estudios han mostrado buenos resultados para los xenoinjertos. ${ }^{29-35}$ En este caso se utilizó un xenoinjerto de hueso bovino.

La segunda gran discusión respecto a los injertos sinusales es el uso o no de las membranas o barreras de regeneración ósea. La mayoría de autores señalan la necesidad de membranas (en especial las no reabsorvibles) para una correcta regeneración ósea. 1, 2, 7-9, 15, 18, 19 Algunos, sin embargo, cuestionan su utilidad 20. En el caso reportado no se usó membrana o barrera de regeneración ósea.

\section{Conclusiones}

El tratamiento del paciente edéntulo parcial en la región posterior del maxilar es un reto para el implantólogo oral, principalmente por la deficiente cantidad (altura y grosor) y calidad de hueso. Esto se debe a un proceso de reabsorción del reborde alveolar y neumatización del seno maxilar tras la exodoncia de dientes en esa región.

Un correcto diagnóstico, apoyado con exámenes auxiliares adecuados (TAC), y planificación del tratamiento son los grandes pilares para el éxito de la rehabilitación en este tipo de casos.

El uso del sistema Dentium Dask para el abordaje lateral del seno maxilar permite desarrollar un procedimiento quirúrgico simplificado para el acceso al antro del seno maxilar a través del abordaje lateral, lo que reduce el potencial de perforación de la membrana del seno y las complicaciones posteriores cuando los materiales del injerto y los implantes dentales se colocan en el seno.

\section{Referencias bibliográficas}

1.- Misch C. Implantología Contemporánea. 3ra. Barcelona: Misch C, Resnik R, Misch-Dietsh F; 2008. Capítulo 6, Anatomía, patología y cirugía de injerto en el seno maxilar; p. $905-974$. 
2.- Ranalli O. La implantología del nuevo milenio. 3ra. Buenos Aires: El Fénix, 2002; p. 219 - 228.

3. Sharan A, Madjar D. Maxillaty sinus pneumatization following extractions: a radiographic study. The International Journal of Oral and Maxillofacial Implants. 2008; 23(1): 48 - 55 .

4.- Ten Bruggenkate C, Van Den Bergh A. Elevacao do soalho do seio maxilar: um procedimento pré-portético valioso. Periodontología 2000. 2005; 5: 176-182.

5.- Hernández F. Injertos óseos en implantología, técnicas y aplicaciones clínicas. Barcelona: Quintessence, 2006; p.133 - 144 .

6.- Woo I, Le B. Maxillary sinus floor elevation: review of anatomy and two techniques. Implant Dentistry. 2004; 13(1): 28 - 32 .

7.- Peńarrocha M. Implantología Oral. Barcelona: Ars Médica, 2001; p. 96 $-113$.

8.- Palti A, Steigmann M. Long - term succes with sinus elevation - criteria and parameters. The International Magazine of Oral Implantology. 2003; 4: $20-24$.

9.- Baladrón J, Colmenero C, Elizondo J, et al. Cirugía avanzada en implantes. Madrid: Ergon, 2000: p. $174-213$.

10.- Gonzáles R, Navla L, Muñoz M, et al. Cirugía preprotésica e implantológica en pacientes con atrofia maxilar severa. Med Oral Patol Oral Cir Bucal. 2005; 10:343-54.

11.- Muñoz C, Romero M, Vallecillo M. Cinco casos de elevación del seno maxilar con autoinjertos. Estudio clínico, radiológico y análisis con MEB. RCOE. 2002; 7(2): 25 -32 .

12.- Barboza F, Lenharo A, eds. Terapia clínica avanzada en implantología. Sao Paulo: Artes Médicas: Vasconcelos L, Francischone C, Kuabara M, Piva L; 2005. Capítulo 8, Injertos en el Seno Maxilar; 145 - 163.

13.- Bottino M. Nuevas Tendencias 4: periodoncia. Sao Paulo: Artes Médicas, 2008; p. $219-223$.

14.- Block M. Atlas en color de cirugía implantológica dental. Madrid: Médica Panamericana, 2002; p. 85 $-101$.

15.- Ranalli O, Ranalli S. Atlas Práctico de Implantología. Santa Fe, el autor, 2008; p. $239-270$.
16.- Chiapasco M, Romeo E. Rehabilitación Implantosoportada en casos complejos. Bogotá: Amolca, 2006; p. $271-298$

17.- Pazos I. Implantología basada en la "calidad total". Madrid: Avances Médicos Dentales, 2002; p. 65 69.

18.- Sekine H, Taguchi T, Seta Sh, Takano M, Takeda T, Kakizawa T. Dental Implant Treatment with different techniques for sinus floor elevation - A case report. Bull Tokyo Dent Coll. 2007; 48(2): 87-91.

19.- Barrachina M, Cabello G, Olmos G, Gonzáles D. Tratamiento Implantológico de la zona posterior del maxilar superior. Elevación del seno maxilar. RCOE. 2002; 7(1): 91-100.

20. Winter A, Pollack A, Odrich R. Placement of implants in the severely atrophic posterior maxilla using localized management of the sinus floor: a preliminary study. The International Journal of Oral and Maxillofacial Implants. 2002; 17(5): $687-95$.

21.- Diaz A, Martinez A. Elevación interna de seno maxilar tras la colocación de implantes BICON oseointegrados. Una alternativa quirúrgico - protésica. Revista CES Odontología. 2001; 14 (1): 49-54.

22. Soltan M, Smiler D. Trephine bone core sinus elevation graft. Implant Dentistry. 2004; 13(2): 148 - 52 .

23. Benner K, Bauer F, Heuckmann K. Balloon-Lift-Control (BLC): a minimal-invasive system for the elevation of the sinus floor mucosa. International magazine of oral implantology. 2007; 8(1): 26- 29.

24. Marx R, Garg A. A novel aid to elevation of the sinus membrane for the sinus lift procedure. Implant Dentistry. 2002; 11(3): $268-71$.

25.- Galindo P, Ávila G, Moreno I, Ríos F, O’Valle F, Mesa F. Descripción de una técnica simplificada para elevación de seno. Periodoncia y Osteointegración. 2008; 18(2): 133 $-138$.

26. Engelke W, Zirkler HU. A new sinuslift generation: keyhole technique - Case report using a new resorbable membrane. Internacional magazine of oral implantology. 2005; 4: $18-21$.

27.- Wainwrigth $\mathrm{M}$, Troedhan $\mathrm{A}, \mathrm{Ku}$ rrek A. The IntraLIft: A new minimal invasive ultrasonic technique for sinus grafting procedures. Inter- national magazine of oral implantology. 2007; 8(3): 30 - 34.

28. Schlegel A, Hamel J, Wichmann M, Either S. Comparative clinical results after implant placement in the posterior maxilla with and without sinus augmnetation. The International Journal of Oral and Maxillofacial Implants. 2008; 23(2): $289-98$.

29. Andreana S, Cornelini R, Edsberg L, Natiella J. Maxillary sinus elevation for implant placement using calcium sulphate with and without DFDBA: six cases. Implant Dentistry. 2004; 13(3): $270-7$.

30. Mangano C, Scarano A, Perrotti V, Lezzi G, Piattelli A. Maxillary sinus augmentation with a porous synthetic hydroxyapatite and bovine - deriver hydroxyapatite: a comparative clinical and histologic study. The International Journal of Oral and Maxillofacial Implants. 2007; 22(6): 980-6.

31. Scarano A, Degidi M, Iezzi G, Pecora G, Piattelli M, Orsini G, et al. Maxillary sinus augmentation with different biomaterials: A comparative histologic and histomorphometric study in man. Implan Dentistry. 2006; 15(2): 197-207.

32. Steigmann M, Garg A. A comparative study of bilateral sinus lift performed with platelet - rich plasma alone versus alloplastic graft material reconstituted with blood. Implant Dentistry. 2005; 14(3): $261-6$

33. Gelbart M, Friedman R, Burlui V, Rohrer M, Atkinson B. Maxillary sinus augmentation using a peptide-modified graft material in three mixtures: a prospective human case series of histologic and histomorphometric results. Implant Dentistry. $2005 ; 14(2): 185-93$.

34. Graziani F, Ducci F, Tonelli M, Askay A, Monier M, Gabriele M. Maxillary sinus augmentation with platelet-rich plasma and fibrinogen cryoprecipitate: a tomographic pilot study. Implant Dentistry. 2005; 14(1): $63-9$

35. Degidi M, Scarano A, Iezzi G, Orsini G, Perrotti V, Strocchi R, et al. Maxillay sinus augmentation using a synthetic cell-binding peptide: a histologic and transmission electron microscopy case study in man. Implant Dentistry. 2005; 14(4): 371- 7 .

Odontol. Sanmarquina 2011; 14(2):21-24 